\title{
Electrophysiological Effects on Renal Ischemia/Reperfusion-Induced Cardiac Hypertrophy
}

\author{
Karine Panico, Giovanni L.Weber, Marcela S. Carneiro-Ramos, João Salinet \\ Universidade Federal do ABC, Santo André, Brazil
}

\begin{abstract}
Acute kidney injury has been previously shown to develop cardiac hypertrophy $(\mathrm{CH})$ also by catecholamines release. This study aims to investigate the influence of sympathetic nervous system (SNS) on the $\mathrm{CH}$-induced by ischemic renal (I/R) injury. Methods: Mice $(C 57 B / / 6)$ were divided in 4 groups: Sham, I/R, Sham atenolol (ATE) and I/R ATE. Morphometric conditions were evaluated and ECG collection was performed using in-house custom written system (hardware and platform). Heart Rate, $R$-R interval, R-peak amplitude, QT interval and QTc (Mitchell's QTcorrection) were manually inspected and calculated by LabChart (V8.1.5, AD Instruments). Results: CH was induced by I/R after $12 d$ when compared to sham ( $p<0.05)$. ATE prevented $\mathrm{CH},(p<0.05)$. I/R induced an increase on $Q T$ interval I/R vs Sham, $p<0.05)$. I/R had $30 \%$ of $R$-peak reduction (vs. Sham) whereas in the treated group it increased 3\% (I/R ATE vs Sham ATE). R-peak also had increase $8 \%$ at the $I / R$ group (vs. $I / R$ ATE). Sham's $R-R$ interval (78,8 $\pm 1,6 \mathrm{~ms})$ and $Q T c(158,1 \pm 15,6 \mathrm{~ms})$ had not differences when compared respectively with the other groups. Conclusion: I/R developed $\mathrm{CH}$ with morphology and electrophysiology changes.
\end{abstract}

\section{Introduction}

Heart and kidneys share several functions in maintaining homeostasis [1]. Acute kidney injury is defined as a decrease in renal function and may be an inflammatory disease that can control other organs such as heart [2]. There are important roles of inflammatory cytokines in the molecular pathways that initiate cardiac hypertrophy [3]. The persistence of abnormal physiological conditions due to cardiovascular diseases eventually gives rise to heart failure (HF) and the inability of the heart to properly pump the blood. The characteristic that precedes $\mathrm{HF}$ is pathological cardiac hypertrophy $(\mathrm{CH})$, defined as an increase in heart size [4]. Pathologic $\mathrm{CH}$ is characterized by increased cardiomyocyte size, increased protein synthesis, re-expression of fetal genes and change in fatty acids to produce glucose as a source of energy Before HF, the hypertrophied heart is considered a compensatory mechanism against an overload of pressure or increased volume, which may allow adequate pumping function for many years, but favoring electrical disturbances in the myocardium [5]. Dysfunctions of the sympathetic nervous system (SNS) are also on other factor, develop of $\mathrm{CH}$ resulting from sympathetic activation to the heart and kidneys. Thus, as catecholamines are released into the circulation, it acts on cardiac tissue through beta adrenergic receptors promoting an increase in cardiac mass [6]. Further, the SNS is able of modulating immune system actions, under different experienced conditions, through the increase, number of defense cells and consequent, increased immune response.

Although echocardiography has been established the "golden standard" in HF, ECG analysis has provided valuable insights on the various electrocardiographic features allowing distinguish wildtype or transgenic mice undergoing different protocols [5]. Among the markers, the QT segment representing the duration of ventricular depolarization and repolarization and the corrected QT interval QTc [7], that the prolongation of the corrected QT interval (QTc) has been associated to congestive $\mathrm{HF}$, electrolyte abnormalities and $\mathrm{HC}$, because these ECG markers are related to the increase of the thickness of the walls and the size of the ventricle under $\mathrm{HC}$ conditions [8]. The peak $\mathrm{R}$ is responsible for the ventricular electrical activity, and this is also one of the markers. The present study aims to investigate a participation of the SNS in the development of $\mathrm{CH}$ during I/R and reperfusion model.

\section{Methods}

\subsection{Protocol}

All procedures were approved by the ethics committee of the Federal University of ABC (Protocol 029/2013). 
Male mice C57B1 / $6(\mathrm{n}=45)$ underwent left renal pedicle occlusion surgery for 60 minutes followed by reperfusion (I / R) for 12 days. Another group of animals, in addition to the surgical procedure, received atenolol treatment (10mg / kg / day) for 5 days from the 7 th day of reperfusion (I/R or Sham ATE group).

\subsection{Morphometric Analysis}

Morphometric conditions were evaluated by:

- Heart weight (HW) and body weight (BW):

$$
\left(\frac{H W}{B W}\right) * 1000
$$

- $\quad$ HW and tibia length (TL)

$$
\left(\frac{H W}{T L}\right) * 100
$$

Both indices indicate the development and installation of cardiac hypertrophy.

\subsection{Electrophysiological Analysis}

ECG collection were performed using in-house custom written system (hardware and platform) with a surgically electrode piercing placed at the DI position (Figure 1). Signals were sampled at $1 \mathrm{kHz}$ and 2-seconds segments analyzed in for off-line. The ECGs were band-pass filtered $(0.5-250 \mathrm{~Hz})$ with a tenth-order zero-phase delayed Butterworth filter. Heart Rate (ms), R-R interval (ms), Rpeak amplitude $(\mathrm{mV})$, QT interval. QTc was calculated by Mitchell's QT-correction.

$$
Q T_{C}=\frac{Q T}{\sqrt{\frac{(R R)}{100}}}
$$

All electrocardiographic markers were automatic calculated by in-house custom codes, based on previously validated algorithms [9] and manually inspected. These results were compared with those calculated by LabChart (V8.1.5, AD Instruments), assumed here as "golden standard".

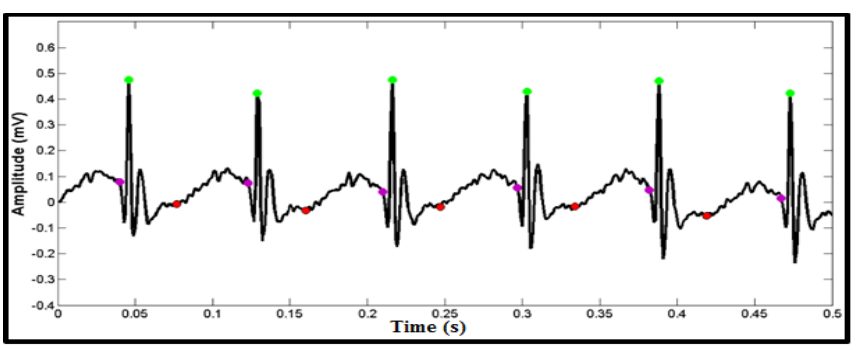

Figure 1: Example of an acquired ECG at DI.

\subsection{Statistics}

The results were submitted to the test of variance (ANOVA) with subsequent Bonferroni test, considering as significant $\mathrm{p}<0.05$. Data expressed as mean \pm standard deviation.

\section{Results}

\subsection{Evaluation of cardiac tropism}

First, we evaluated the kidney weight $(\mathrm{KW})$ to $\mathrm{BW}$ ratio in all experimental groups (see Figure 2). As expected, there was a significant decrease in the of left kidney weight (LKW) to BW ratio compared to their respective Sham group during the 12 days of reperfusion, and the LKW compared to right kidney weight (RKW) in the same groups, indicating that there was a renal injury. A significant and compensatory increase of the RKW was observed in relation to the respective Sham group. We also observed an improvement in the LKW treated with atenolol, suggesting a reno-protective function (Figure 2).
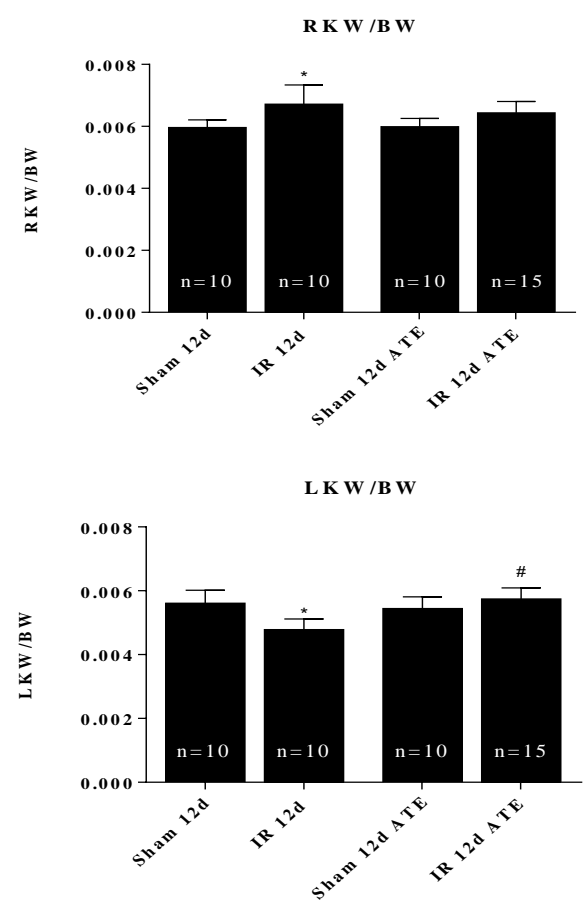

Figure 2: Upper: Ratio between RKW and BW (mg / g) in the different experimental groups. * Vs Sham $\mathrm{p}<0.05$; Lower) ratio between $\mathrm{LKW}$ and $\mathrm{BW}(\mathrm{mg} / \mathrm{g})$ in the different experimental groups. * Vs Sham $\mathrm{p}<0.05$; \# Vs IR $\mathrm{p}<0.05$.

With the renal lesion confirmed, we analyzed it impact on the cardiac hypertrophy development.

We observed a significant increase in the $\mathrm{HW}$ and BW ratio after 12 days of reperfusion, evidencing a mass gain 
of cardiac tissue, suggesting the establishment of the hypertrophic condition (Figure 3 upper). Animals receiving atenolol showed a prevention of hypertrophy when compared to the respective Sham group. Furthermore, the HW to TL ratio (Figure 3 lower) was also increased in $\mathrm{I} / \mathrm{R}$ animals.

H W /B W

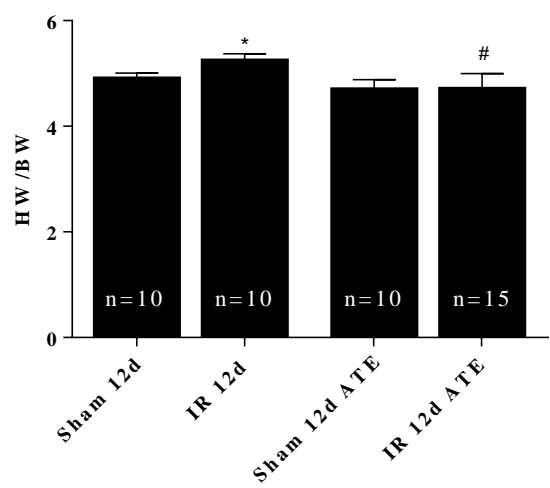

H W / T L

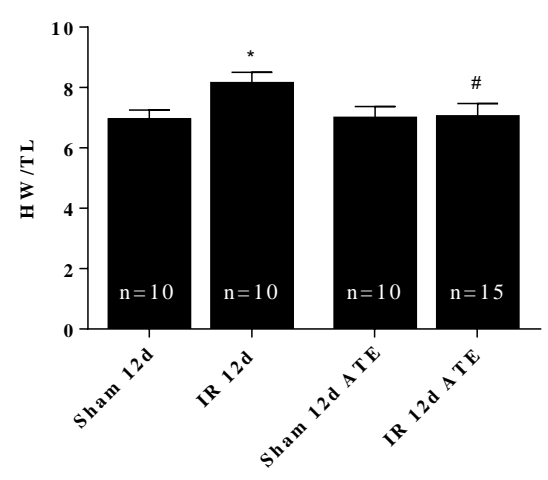

Figure 3: (Upper) Ratio between $\mathrm{HW}$ and BW (mg / g) in the different experimental groups. * Vs Sham $\mathrm{p}<0.05$; \# Vs I / R p <0.05; (Lower) ratio between HW and TL (mg / $\mathrm{cm})$ in the different experimental groups. ${ }^{*}$ vs Sham $\mathrm{p}$ $<0.05$; \# Vs I/R p $<0.05$.

\subsection{Induced Electrophysiological by HC}

We also evaluated the cardiac electrical profile of the animals at 4 different times during the reperfusion period, before and after the treatment with atenolol (Figure 4).

The measurements were performed on days 1, 5, 8 and 12, times previously investigated by our group where we showed important events during the development of cardiac hypertrophy.

Renal ischemia protocol induced an increase on QT interval after $5 \mathrm{~d}$ of reperfusion. I/R had $30 \%$ of R-peak reduction compared to Sham group whereas in the treated group it increased 3\%. R-peak also had increase $8 \%$ at the
I/R group compared to I/R ATE group. Sham's R-R interval $(78,8 \pm 1,6 \mathrm{~ms})$ and QTc $(158,1 \pm 15,6 \mathrm{~ms})$ had not shown statically significance when compared respectivally with the other groups.

A)

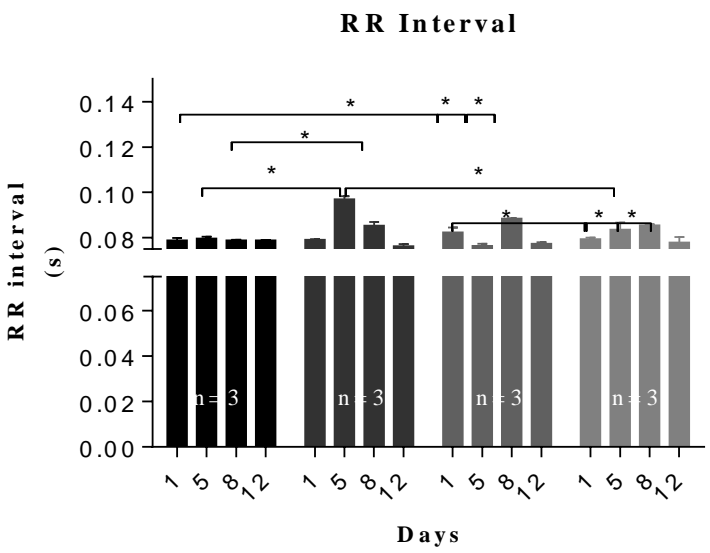

B)

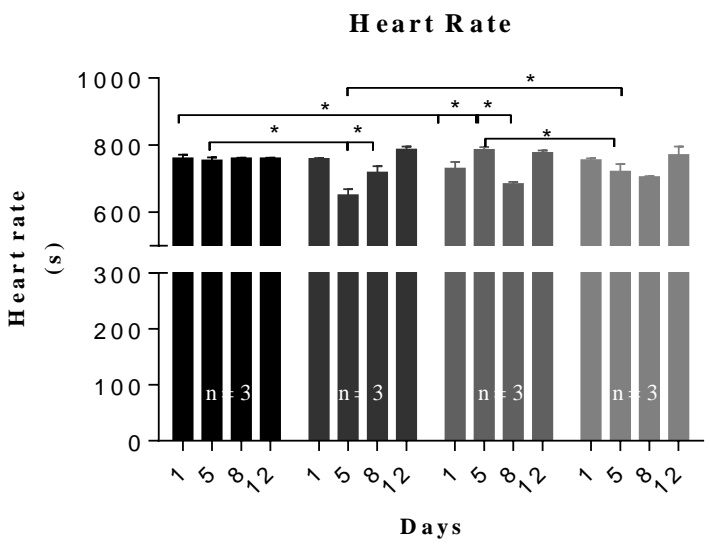

C)

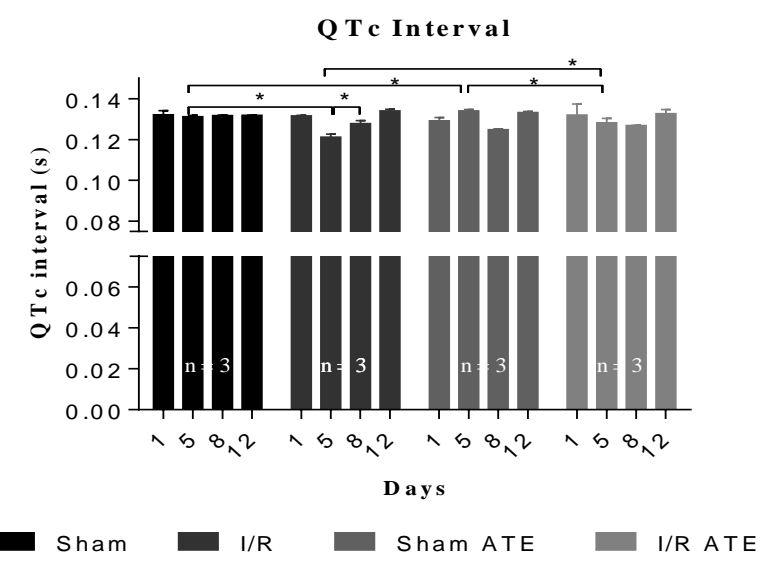


Figure 4: Electrocardiographic parameters in the different experimental groups. A) RR interval; B) heart rate; and C) Interval QTc. Groups: Sham, I / R, Sham treated and I / R treated with atenolol.

\section{Conclusion}

The renal ischemia and reperfusion model was performed adequately, as expected, generating a picture of left kidney injury. Moreover, renal ischemia and reperfusion was able to induce cardiac hypertrophy in animals at 12 days of reperfusion. Cardiac hypertrophy was accompanied by a decrease in response to treatment (ATE) suggesting an increase in cardiac sensitivity to SNS. The experimental $\mathrm{I} / \mathrm{R}$ protocol was able to increase the QT interval after 5 days of reperfusion.

Renal ischemia and reperfusion increased the peak $R$ amplitude compared to its respective atenolol treated group.

\section{Acknowledgements}

We thank the follow agencies that contributed to this study: FAPESP; CNPq and UFABC.

\section{References}

[1] Ronco, Claudio, Mikko Haapio, Andrew A. House, Nagesh Anavekar, and Rinaldo Bellomo. "Cardiorenal Syndrome." Journal of the American College of Cardiology 52, no. 19 (November 4, 2008): 1527-39.

[2] Ronco, Claudio, Chang-Yin Chionh, Mikko Haapio, Nagesh S. Anavekar, Andrew House, and Rinaldo Bellomo. "The Cardiorenal Syndrome." Blood Purification 27, no. 1 (2009): 114-26.

[3] Ang, Dsc, and Cc Lang. "The Prognostic Value of the ECG in Hypertension: Where Are We Now?" Journal of Human Hypertension 22, no. 7 (July 2008): 460-67.

[4] Parsa, Hesam, Bryan Z. Wang, and Gordana Vunjak Novakovic. "A Microfluidic Platform for the High-Throughput Study of Pathological Cardiac Hypertrophy." Lab on a Chip 17(19):3264-3271.

[5] Sysa-Shah, Polina, Lars L. Sørensen, M. Roselle Abraham, and Kathleen L. Gabrielson. "Electrocardiographic Characterization of Cardiac Hypertrophy in Mice That Overexpress the ErbB2 Receptor Tyrosine Kinase." Comparative Medicine 65, no. 4 (August 2015): 295-307.

[6] Barry, Sean P., Sean M. Davidson, and Paul A. Townsend. "Molecular Regulation of Cardiac Hypertrophy. The International Journal of Biochemistry \& Cell Biology 40, no. 10 (2008): 2023-39.

[7] Mitchell, G. F., A. Jeron, and G. Koren. "Measurement of Heart Rate and Q-T Interval in the Conscious Mouse." The American Journal of Physiology 274, no. 3 (March 1998): H747751.

[8] Cheng, Y., X. Wan, T. A. McElfresh, X. Chen, K. S. Gresham, D. S. Rosenbaum, M.P. Chandler, and J. E. Stelzer. Impaired contractile function due to decreased cardiac myosin binding protein C content in the sarcomere. American Journal of Physiology Heart and Circulatory Physiology 305 (1)(2013):H52-H65.

[9] Salinet, J. L., J. P. V. Madeiro, P. C. Cortez, P. J. Stafford, G. André Ng, G. André Ng, and F. S. Schlindwein. "Analysis of QRS-T Subtraction in Unipolar Atrial Fibrillation Electrograms." Medical \& Biological Engineering \& Computing 51, no. 12 (December 2013): 1381-91.

Address for correspondence.

Name. Karine Panico

Biosistemas

CCNH - Universidade Federal do ABC

Santo André, São Paulo, Brazil

E-mail:. karipan84@gmail.com 